\title{
Blockchain-based Electronic Health Record System for efficient Covid-19 Pandemic Management
}

\author{
Faheem Ahmad Reegu ${ }^{1}$, Salwani Mohd Daud ${ }^{2}$, Shadab Alam ${ }^{3 *}$, Mohammed Shuaib 4* \\ 1, 2,4 Razak faculty of technology and Informatics, Universiti Teknologi Malaysia \\ ${ }^{3}$ Department of computer science, Jazan University, Jazan \\ *Corresponding author: Shadab Alam, Department of computer science, Jazan University, Jazan, Email: \\ s4shadab@gmail.com
}

\begin{abstract}
Electronic Health Record (EHR) is being used in most healthcare institutions to preserve and share health records instead of a paper-based method. Data records must be exchanged amongst various parties and users' privileges to manage access to their records should also be provided. In addition to the basic standards of secrecy, confidentiality and integrity of information, these facts further demonstrate the need for interoperability and consumer control to access their personal data. Electronic Health Record (EHR) system faces issues of protection of data, trust and management issues. In recent Covid-19 pandemic, various applications, tools and websites were launched that stores records. Also, many personal records related to health need to be shared among different parties for early detection, contact tracing, monitoring and the future prediction that requires accurate and reliable data. Simultaneously, the citizens will be hesitant in providing their personal details due to privacy concerns and social stigma. Blockchain technology has arisen as a powerful technology that can offer the immutability, confidentiality and user access properties of stored information and provided distributed storage. This paper analyses the blockchain suitability in EHR and its further applications in efficient Covid-19 pandemic management
\end{abstract}

Keywords: Electronic Health Record, EHR, Blockchain, Covid-19

\section{Abbreviations}

COVID-19: Coronavirus Disease 2019; EHR- Electronic Health Record; health information exchanges (HIE)

\section{Introduction}

Electronic health record (EHR) is a real-time official health record of a patient in a digital version that can be shared easily, securely, and instantly among different facilities, and the departments. It includes every data required to get the details of the patient, including medical history, radiology images, diagnoses, medications, immunisation dates, treatment plans, allergies, laboratory results, etc. It plays a significant role in healthcare because it delivers easy access to health records used in decision making related to the patient's care. Deficiency of standardisation and regulation of sharing files create EHR interoperability is an important issue that has to be considered by healthcare providers [1]. The issues of determining confidentiality and security during information sharing increase the complication in attaining interoperability. [2]. EHRs and different other Health Information Technology (HIT) organisations are not organised due to low communication standards throughout distinct EHRs, increased charges of integration, low participation of patients in sharing of data, and Lack of identification of patient throughout the health information exchanges (HIE) [3]. 
A blockchain is defined as a digitised record of the transaction. This technology is titled as blockchains because of its construction. It contains records of the individuals, known as inter-linked blocks and organised in a solitary list, known as a chain. Each transaction is appended to the blockchain after authentication by various inter-connected validating nodes[4], [5]. Blockchain is a particular case of Distributed Ledger Technology (DLT) [6]. This distributed network of computer nodes certifies a solitary system could not add invalid or illegal blocks to the specific chain. Whenever a unique block is supplemented to a blockchain, it is inter-lined with the preceding blocks by using a "cryptographic hash" produced from the insides of the former block[7], [8]. Blockchain is very much suitable for reliable information sharing in EHR due to its features of immutability, use of cryptographic functions for secure communication [9][10], [11]. There are various possible application scenarios of blockchain-based EHR in pandemic management; like contact tracing, data aggregation, data sharing, lab record management, Covid certificate for infected and recovered persons, and prediction for further progress infection population and supply chain management for vaccines and other essentials efficiently and reliably.

\section{Electronic Health Record (EHR)}

In the past, all therapeutic records were documented and reported by using papers and is filed manually. Moreover, these medical records were retrieved and filed from specifically designed shelves to carry these records' file folders. After the advent of information technology, things started to revolutionise, and records were converted into a digital medium for storage and retrieval that gave birth to electronic healthcare records systems [12] [13].

Automated management for the records makes the patient records accessible that can be linked easily to the monitoring gadgets for recording and interpreting patients' data in the EHR [14]. Rapidly with the growing period, the EHRs started to use in production and in enormous amounts to maintain the medical data that were found to be effective in doing research related to epidemiology [14].

\section{EHR Requirements}

EHR requirements are as follows:

a) Interoperability: Interoperability in EHR is described as the degree to which devices and systems can transfer data and translate the shared data [15] [16].

b) Privacy and security: security and privacy in the healthcare environment are intended to give patients the authority to manage their medical records through the provision of authorisations [17] [18].

c) Confidentiality: confidentiality is distinguished from privacy related to the dimension of reliable communication or contract between providers and the patients. Patient's records need to be held in confidence [18], [19].

d) Access control: Medical information should then be accessible only to authorised healthcare specialists and patients. Patients should obtain their data and provide control over who can access it [20].

e) Data sharing: exchange of medical records is an essential requirement with the patient's treatment being scattered over various health care providers; therefore, the data is shared with other medical institutions and government [20].

f) Data Integrity and Availability: integrity implies preserving the efficiency and consistency of the data. In EHR, it leads to the fact that data has not been damaged by unapproved use [21].

\section{Issues in EHR}

EHR technology is resolving some issues on one side but further create some issues on another side. It becomes easy to hack the data if the prior precautions have not been taken. As it is a new way of storing data, staff need proper training to use it. Electronic health records contain highly crucial and critical patient-related medical data, requiring safe storage, sharing, retrieval, and scope. To encourage and enhance healthcare facilities' condition, medical knowledge must be preserved and exchanged routinely by several different shareholders, including physicians, healthcare professionals, 
pharmacies, insurance agencies, and researchers. Overall, individual key data exchange styles require rigorous confidentiality and accountability requirements during data transfers [22].

\section{Blockchain in EHR}

Blockchain can modernise the electronic health record exchanges by delivering a secure approach for medical data exchange of medical data in the field of healthcare productiveness, by safeguarding it through a dispersed peer-to-peer linkage. The Blockchain approach is proposed to provide sustenance and comfort to the procedure for understanding the dispersed technology of ledger [23]. Advances in this innovative blockchain technology have steered to provide transactions in a better way, including insurance billing, health records, and smart contracts. One main advantage of using blockchain technology in healthcare is that it can include record interoperability, improved patient information access, and system monitoring spanning a device's entire life cycle in the blockchain substructure. Blockchain can be used along with other emerging technologies like the Internet of Things (IoT) and Cloud computing for better EHR solutions[24]-[26]. Blockchain will handle the security, reliability, immutability and interoperability features[27]-[29]. Access to health histories of patients is vital to recommend medicines, with blockchain appropriately. It will be easy to improve the framework of medical care services radically[30].

\section{Blockchain Application in the pandemic situation:}

The potential utilisation of the blockchain system to control and mitigate the COVID-19 scenario is discussed and examined in detail in the following sections.

\subsection{Clinical Trial Data Management}

Clinical trials should maintain data according to regulations such as records to be open to stakeholders, privacy and record protection, and immutability [31]. Blockchain technology will enable clinicians and doctors to record health real-time information and make it available. It improves the accuracy of the information and facilitates the exchange of data and ensures compliance and offers an audit trail for better privacy and data protection [32][33]

\subsection{Vaccine and essential medicine supply chain}

Blockchain can efficiently manage the health supply chain, particularly in pandemic situations involving significant global cross-border transactions. There can be instability in its delivery until the licensed version of the vaccine is available for sales and marketing. Corrupt practices such as false vaccines, over-pricing, stock accumulating, etc., may be feasible. Using the blockchain-based medical supply chain, these problems can be handled efficiently [34].

\subsection{Contact tracing}

Governments and health facilities are active in the patient contact-tracking systems, but the records obtained can be misused. The use of blockchains will provide consistency and reliability of data. Blockchain networks can track patient activity and provide real-time updates to areas affected [35]. Further, the records can generate a report for infected and possible infected population, based on contacts.

\subsection{Data Aggregation}

Sorting, collecting and acquiring the information needed to track the outbreak, deciphering trends, and administering experiments are vital resources to respond effectively to the pandemic. Blockchain's ability to verify and retain permanent, real-time information ensures data integrity [36]. Using a blockchain network provides monitoring and communications infrastructure to help capture, store and analyse virus dissemination and containment data.

\subsection{User Data Privacy}

Policymakers and healthcare practitioners need to obtain patient data by patent monitoring and other initiatives for improved decision-making concurrently, patient privacy and confidentiality issues also need to be discussed. During these worrying days, a balancing act between record management and user privacy management needs to be carried out to 
improve trust in the system. Blockchain is a potential solution to capture and display patient information, screen patient procedures, and establish social isolation degrees while preserving privacy.

6.6 Early detection of vulnerable population

Various AI-based triage systems potentially alleviate patient anxiety. The online bot will help understand the initial signs of early detection and then direct them by preventive measures such as social distancing, hand hygiene, etc. If symptoms increase, alert users to medical care facilities [37] [39]. Patient information's confidentiality and privacy are of the utmost importance for the security of their personal and social values. The blockchain-based framework can effectively handle these security and privacy issues [38].

\section{Discussion and Conclusion}

EHR is the digital record of the medical history of the patient. It has solved many issues related to data handling and its security. This paper has reviewed the various EHR requirements, issues with EHR systems, and use of blockchain to resolve these issues. We have further examined the different possible application scenarios of blockchain-based EHR in pandemic management; like contact tracing, data aggregation, data sharing, user data privacy, Covid certificate for infected and recovered persons, prediction for further progress of infection population and supply chain management for vaccines and other essential commodities efficiently and reliably. Blockchain is a technology that can be efficiently used to counter the shortcomings of traditional EHR issues and to efficiently and effectively help in managing the Covid-19 pandemic issue by providing reliable, accurate and secure data storage and exchanges.

\section{Bibliography}

[1] O'Dowd, "Lack of EHR Interoperability Standards Challenges Health IT," 2017. .

[2] S. Bhartiya, D. Mehrotra, and A. Girdhar, "Issues in Achieving Complete Interoperability while Sharing Electronic Health Records," Elsevier, 2016, doi: 10.1016/j.procs.2016.02.033.

[3] G. Barrick, "4 Reasons Why EHR Interoperability is a Mess (and How to Fix It) | Datica," Datica , 2019. .

[4] S. T. Siddiqui, R. Ahmad, M. Shuaib, and S. Alam, "Blockchain Security Threats, Attacks and Countermeasures," in Advances in Intelligent Systems and Computing, 2020, vol. 1097, pp. 51-62, doi: 10.1007/978-981-15-1518-7_5.

[5] M. Shuaib, S. M. Daud, S. Alam, and W. Z. Khan, "Blockchain-based framework for secure and reliable land registry system," Telkomnika (Telecommunication Comput. Electron. Control., vol. 18, no. 5, pp. 2560-2571, Oct. 2020, doi: 10.12928/TELKOMNIKA.v18i5.15787.

[6] T. Hardin and D. Kotz, "Blockchain in Health Data Systems: A Survey," 2019 6th Int. Conf. Internet Things Syst. Manag. Secur. IOTSMS 2019, pp. 490-497, 2019, doi: 10.1109/IOTSMS48152.2019.8939174.

[7] M. Shuaib, S. Alam, S. Mohd, and S. Ahmad, "Blockchain-Based Initiatives in Social Security Sector," 2020.

[8] S. T. Siddiqui, S. Alam, and M. Shuaib, "Cloud Computing Security using Blockchain," J. Emerg. Technol. Innov. Res., vol. 6, no. August, 2019, [Online]. Available: www.jetir.org.

[9] S. T. Siddiqui, M. Shuaib, A. K. Gupta, and S. Alam, "Implementing Blockchain Technology : Way to Avoid Evasive Threats to Information Security on Cloud," in 2020 International Conference on Computing and Information Technology, 2020, no. October, pp. 87-91.

[10] W. J. Gordon and C. Catalini, "Blockchain Technology for Healthcare: Facilitating the Transition to Patient-Driven Interoperability," Comput. Struct. Biotechnol. J., vol. 16, pp. 224-230, 2018, doi: 10.1016/j.csbj.2018.06.003.

[11] C. Catalini and J. S. Gans, "Some Simple Economics of the Blockchain," SSRN Electron. J., Nov. 2016, doi: 10.2139/ssrn.2874598.

[12] G. Marquez, "The history of electronic health records | Elation Health | Clinical First Electronic Health Record | Elation Health," Elation Health, 2017. .

[13] J. Atherton, "Development of the electronic health record," Virtual Mentor, vol. 13, no. 3, pp. 186-189, 2011, doi: 10.1001/virtualmentor.2011.13.3.mhst1-1103.

[14] R. S. Evans, "Electronic Health Records: Then, Now, and in the Future," Yearb. Med. Inform., no. Suppl 1, pp. S48S61, May 2016, doi: 10.15265/IYS-2016-s006.

[15] E. Adel, S. El-Sappagh, S. Barakat, and M. Elmogy, "Distributed electronic health record based on semantic 
interoperability using fuzzy ontology: a survey," Int. J. Comput. Appl., vol. 40, no. 4, pp. 223-241, 2018, doi: 10.1080/1206212X.2017.1418237.

[16] U. Shrivastava, J. Song, and B. Han, "Teh implications of patient data security considerations for EHR interoperability and downtime recovery," 2019.

[17] C. S. Kruse, B. Smith, H. Vanderlinden, and A. Nealand, "Security Techniques for the Electronic Health Records," J. Med. Syst., vol. 41, no. 8, 2017, doi: 10.1007/s10916-017-0778-4.

[18] W. Bani Issa et al., "Privacy, confidentiality, security and patient safety concerns about electronic health records," Int. Nurs. Rev., pp. 1-13, 2020, doi: 10.1111/inr.12585.

[19] Valerie S and Prater, "Confidentiality, privacy and security of health information: Balancing interests - Health Informatics Online Masters | Nursing \& Medical Degrees," 2014..

[20] C. A. Ardagna, S. De Capitani Di Vimercati, S. Foresti, T. W. Grandison, S. Jajodia, and P. Samarati, "Access control for smarter healthcare using policy spaces," Comput. Secur., vol. 29, no. 8, pp. 848-858, 2010, doi: 10.1016/j.cose.2010.07.001.

[21] Y. Y. Chen, J. C. Lu, and J. K. Jan, "A secure EHR system based on hybrid clouds," J. Med. Syst., vol. 36, no. 5, pp. 3375-3384, 2012, doi: 10.1007/s10916-012-9830-6.

[22] P. ; Palvia, T. ; Jacks, and W. Brown, "Critical Issues in EHR Implementation: Provider and Vendor Perspectives," Commun. Assoc. Inf. Syst., vol. 36, pp. 707-725, 2015, doi: 10.17705/1CAIS.03636.

[23] A. H. Mayer, C. A. da Costa, and R. da R. Righi, "Electronic health records in a Blockchain: A systematic review," Health Informatics J., vol. 26, no. 2, pp. 1273-1288, 2020, doi: 10.1177/1460458219866350.

[24] A. Samad, M. Shuaib, and M. Rizwan Beg, "Monitoring of Military Base Station using Flooding and ACO Technique: An Efficient Approach," Int. J. Comput. Netw. Inf. Secur., vol. 9, no. 12, pp. 36-44, Dec. 2017, doi: 10.5815/ijcnis.2017.12.05.

[25] S. T. Siddiqui, S. Alam, R. Ahmad, and M. Shuaib, "Security threats, attacks, and possible countermeasures in internet of things," in Lecture Notes in Networks and Systems, vol. 94, 2020, pp. 35-46.

[26] S. Alam, S. T. Siddiqui, A. Ahmad, R. Ahmad, and M. Shuaib, "Internet of things (IoT) enabling technologies, requirements, and security challenges," in Lecture Notes in Networks and Systems, vol. 94, 2020, pp. 119-126.

[27] S. Alam, M. Shuaib, and A. Samad, "A Collaborative Study of Intrusion Detection and Prevention Techniques in Cloud Computing," in Lecture Notes in Networks and Systems, vol. 55, 2019, pp. 231-240.

[28] S. T. Siddiqui, M. Shuaib, and B. Mohammad.Ubaidullah, "Web Based Requirements Management Tools for Software Development : A Study," Proc. 12th INDIACom; INDIACom-2018; IEEE, no. February 2019, pp. 10-15, 2018.

[29] S. Abdus, A. Shadab, S. Mohammed, and B. Mohammad.Ubaidullah, "Internet of Vehicles (IoV) Requirements, Attacks and Countermeasures," 5 Int. Conf. "Co mputing Sustain. Glob. Dev., no. March, pp. 4037-4040, 2018.

[30] S. T. Siddiqui, S. Alam, Z. A. Khan, and A. Gupta, Cloud-Based E-Learning: Using Cloud Computing Platform for an Effective E-Learning, vol. 851. Springer Verlag, 2019, pp. 335-346.

[31] D. R. Wong, S. Bhattacharya, and A. J. Butte, "Prototype of running clinical trials in an untrustworthy environment using blockchain," Nat. Commun., vol. 10, no. 1, pp. 1-8, 2019.

[32] D. G. Glover and J. Hermans, "Improving the traceability of the clinical trial supply chain," Appl. Clin. Trials, vol. 26, no. 11/12, pp. 36-38, 2017.

[33] I. A. Omar, R. Jayaraman, K. Salah, M. C. E. Simsekler, I. Yaqoob, and S. Ellahham, "Ensuring protocol compliance and data transparency in clinical trials using Blockchain smart contracts," BMC Med. Res. Methodol., vol. 20, no. 1, pp. 1-17, 2020, doi: 10.1186/s12874-020-01109-5.

[34] A. Chawla and S. Ro, "Coronavirus (COVID-19)-Is blockchain a true savior in this pandemic crisis," Available SSRN 3655337, 2020.

[35] M. M. Arifeen, A. Al Mamun, and M. Shamim Kaiser, "Blockchain-enable Contact Tracing for Preserving User Privacy During COVID-19 Outbreak," no. July, pp. 1-11, 2020, [Online]. Available: www.preprints.org.

[36] D. S. W. Ting, L. Carin, V. Dzau, and T. Y. Wong, "Digital technology and COVID-19," Nat. Med., vol. 26, no. 4, pp. 459-461, 2020.

[37] T. P. Mashamba-Thompson and E. D. Crayton, "Blockchain and artificial intelligence technology for novel coronavirus disease-19 self-testing," Diagnostics, vol. 10, no. 4. Multidisciplinary Digital Publishing Institute, 2020, doi: 10.3390/diagnostics10040198. 